\title{
Nistagmo infantil: uma condição inócua ou algo mais? A propósito de um caso clínico
}

Elmano Margato, ${ }^{1}$ Rita Sebastião ${ }^{2}$

\section{RESUMO}

Introdução: O nistagmo infantil pode ou não estar associado a alterações visuais e/ou neurológicas e constitui uma entidade incomum e pouco observada na prática clínica em Medicina Geral e Familiar. Procurou-se, assim, perceber como deve o médico de família atuar perante esta condição.

Descrição do caso: Descreve-se o caso de um lactente sem antecedentes pessoais ou familiares de relevo, tendo o médico de família, na consulta de vigilância de saúde infantil e juvenil dos quatro meses, identificado a presença de um nistagmo bilateral, conjugado, simétrico, horizontal e contínuo. Com a rápida orientação para a consulta de oftalmologia e neurologia pediátricas foi possível excluir a existência de patologia neurológica concomitante. A criança manteve seguimento no oftalmologista e no médico de família, com vigilância da sua evolução, de acordo com as recomendações atuais.

Comentário: Este caso clínico demonstra a importância de uma avaliação global e sistematizada das crianças na consulta de saúde infantil e juvenil. Um exame objetivo completo não deve ser descurado, evitando suposições precipitadas. Reforça ainda a necessidade de uma avaliação oftalmológica adequada, particularmente nos primeiros meses de vida - quando a forma infantil do nistagmo habitualmente se manifesta -, bem como a importância de uma investigação adicional perante esta condição. Embora muitos destes casos ocorram de forma isolada, a maioria parece estar associada a defeitos visuais, tendo o médico de família um papel primordial na identificação, encaminhamento e acompanhamento destas crianças, devendo estar atento a eventuais perturbações no seu desenvolvimento e socialização, bem como a consequências na dinâmica e estabilidade familiar.

Palavras-chave: Nistagmo, Congénito; Nistagmo, Patológico; Médico de família.

\section{INTRODUÇÃO}

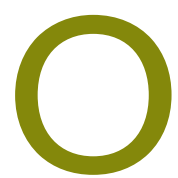

nistagmo é uma entidade clínica caracterizada por oscilações rítmicas e involuntárias dos olhos. Estes movimentos podem ser sacádicos, com uma fase de desvio lento do olhar sucedida de um movimento rápido de reposicionamento do olho na posição primária; pendulares, nos quais ocorre um desvio do olho e posterior retorno à posição inicial de forma mais lenta; ou uma combinação de ambos. ${ }^{1-3}$

Existem diferentes nomenclaturas propostas para caracterizar esta condição. Neste artigo optou-se por uma classificação mais simples, dividindo globalmen-

1. Médico Interno de Medicina Geral e Familiar. UCSP de Sete Rios, ACeS Lisboa Norte.

2. Médica Especialista em Medicina Geral e Familiar. UCSP de Sete Rios, ACeS Lisboa Norte. te o nistagmo em três tipos ${ }^{1,4}$ - fisiológico, ocorrendo em indivíduos saudáveis como resposta a determinados estímulos (e.g., estímulo vestibular, mediante a rotação da cabeça); adquirido, surgindo habitualmente após os 4-6 meses de idade ${ }^{1,5}$ e tipicamente associado a lesões do sistema vestibular, visual ou lesões neurológicas que afetem a integração sensorial; ${ }^{6}$ infantil (anteriormente designado "congénito»), surgindo habitualmente até aos seis meses de idade. ${ }^{5,7}$ Embora este último possa ocorrer de forma isolada (idiopático), é mais frequente a sua associação a um défice visual de base (nistagmo sensorial), ${ }^{8}$ podendo também surgir associado a outras patologias, como estrabismo, albinismo ocular ou oculocutâneo, doenças neurológicas e/ou metabólicas diversas ou integrando um quadro sindromático de uma entidade benigna designada por spasmus nutans (caracterizada clinicamente por uma 
tríade de nistagmo, oscilações rítmicas da cabeça e torcicolo). ${ }^{5}$

O nistagmo infantil, presente desde o nascimento ou, mais comummente, surgindo nos primeiros meses de vida, ${ }^{5,9}$ constitui uma entidade incomum e pouco observada na prática clínica em Medicina Geral e Familiar.

É difícil estimar a prevalência do nistagmo infantil, tendo sido relatados valores muito díspares - desde um caso por 1.000 até um por 500.000 indivíduos. ${ }^{10}$ Alguns estudos apontam para uma incidência a rondar os $0,015 \% .{ }^{9}$ Dada a sua raridade, é normal que o médico de família tenha dúvidas perante uma criança com nistagmo, nomeadamente no que respeita à evolução clínica do quadro, medidas a adotar e precauções a tomar na vigilância destes doentes. Relata-se o caso de uma criança de quatro meses que apresentou um nistagmo numa consulta de vigilância de saúde infantil e juvenil (SIJ). Uma vez que esta condição na infância pode estar associado a múltiplas comorbilidades e dada a necessidade de uma abordagem diagnóstica e/ou terapêutica adequada e atempada do mesmo, coloca-se uma questão pertinente: qual o papel do médico de família perante uma criança com nistagmo? Neste sentido, procurou-se expor de forma breve, clara e à luz da evidência atual, como proceder perante esta entidade clínica nos cuidados de saúde primários, orientando a atuação do médico de família.

\section{DESCRIÇÃO DO CASO}

Lactente, sexo masculino, quatro meses, caucasiano.

Primeiro filho de casal não consanguíneo, inserido numa família nuclear, classe média-alta (classe II de Graffar), estadio II do ciclo de vida de Duvall. História de estrabismo no tio-avô e bisavô paternos, sem outros antecedentes familiares de relevo.

Gravidez espontânea, de baixo risco, devidamente vigiada. Mãe sem antecedentes obstétricos. Diagnóstico pré-natal de comunicação interventricular (CIV) às 28 semanas. Parto de termo, por cesariana devido a paragem de progressão de trabalho de parto, sem outras complicações. Somatometria ao nascer adequada e período neonatal sem intercorrências. Realizou rastreio auditivo, de cardiopatias congénitas e de doenças metabólicas, que foram negativos, tendo sido encaminhado ainda na maternidade para consulta de cardiologia pediátrica devido à CIV.
Foi realizada a primeira consulta de SIJ aos 14 dias de vida, tendo o restante plano de seguimento sido cumprido sem intercorrências. Boa evolução estaturoponderal e do perímetro cefálico; desenvolvimento psicomotor adequado. Apresentava apenas um sopro holossistólico à auscultação cardíaca, consequente à CIV supracitada.

Na quarta consulta de SIJ, com três meses e 26 dias de vida, objetivou-se um nistagmo bilateral, conjugado, simétrico e horizontal, do tipo sacádico com fase rápida para a direita. Este manifestava-se de forma contínua, embora com variações na sua intensidade. Não foram apuradas alterações da motricidade ocular com a fixação do olhar, nem a existência de uma zona nula, isto é, uma determinada posição do olhar na qual o nistagmo remitisse. A criança não apresentou uma posição anómala da cabeça nem esta foi referida pelos progenitores quando inquiridos. Não foi possível avaliar o efeito da convergência ocular por este reflexo ainda não estar presente. Os progenitores ter-se-ão apercebido destes movimentos oculares anormais entre as seis e oito semanas de vida, não tendo, contudo, questionado relativamente a este assunto por desconhecerem esta entidade clínica, tendo interpretado o nistagmo como «curiosidade» do lactente em relação ao meio envolvente.

Apesar da presença de nistagmo, os reflexos pupilares estavam mantidos bilateralmente, a criança dirigia o olhar e fixava estímulos luminosos, sorria e mantinha um contacto sintónico esperado para a sua idade, não aparentando alterações grosseiras da acuidade visual. Apresentava um bom desenvolvimento psicomotor e estaturo-ponderal, sem outras alterações de destaque no exame objetivo, em particular na anatomia ocular visualizável sem instrumentação própria. Uma vez que o nistagmo não apresentava características preocupantes (Tabela 1), ,4-5,5,911-14 os pais foram tranquilizados, tendo-lhes sido explicado o quadro clínico, mas ressalvando que seria necessária uma avaliação oftalmológica mais especializada para excluir, dentro do possível, eventuais alterações patológicas.

A criança foi referenciada para a oftalmologia pediátrica, não tendo sido objetivadas alterações dos meios transparentes do globo ocular ou do fundo ocular. Não se apurou qualquer defeito da visão, tendo sido assumido um nistagmo infantil do tipo idiopático. Por 
TABELA 1. Sintomas e sinais de alerta do nistagmo infantil, sugerindo etiologia não idiopática

Nistagmo infantil - Sintomas e sinais de alerta

Aparecimento logo após o nascimento ou depois dos 4-6 meses de idade.

Nistagmo unilateral, desconjugado ou dissociado.*

Direção de oscilação vertical.

Nistagmo/movimentos oculares atípicos (e.g., nistagmo em dente de serra, alternante periódico, alta frequência de oscilação, opsoclonia, flutter ocular e outros tipos de oscilações sacádicas, apraxia motora ocular).

Oscilópsia. ${ }^{\dagger}$

Presença de nistagmo optocinético fisiológico (tipicamente invertido no nistagmo infantil).

Distúrbios oftalmológicos concomitantes ${ }^{\ddagger}$ (e.g., defeito pupilar aferente, opacificações da córnea e/ou cristalino, défice visual marcado, fotofobia, papiledema, hipoplasia do nervo ótico ou da fóvea).

Sintomas neurológicos concomitantes (e.g., vertigens, cefaleia, vómitos, convulsões, paralisia/paresia das extremidades, ataxia). Atraso do desenvolvimento psicomotor, crescimento estaturo-ponderal e/ou perímetro cefálico anormal para a idade.

História familiar de patologia com manifestações oftalmológicas e antecedentes pessoais de complicações durante a gravidez/parto ou prematuridade marcada.

* Nistagmo desconjugado: direção de oscilação diferente entre os olhos; nistagmo dissociado: oscilações dos olhos com diferentes frequências e/ou amplitudes entre si.

† Ter em atenção que apesar do nistagmo infantil não estar normalmente associado a oscilópsia, 1,5 crianças pequenas com nistagmo adquirido podem não expressar esta queixa. ${ }^{14}$

‡ Notar que alterações oftalmológicas podem não ocorrer isoladas, associando-se a patologia neurológica ou sistémica de base.

sugestão do oftalmologista foi encaminhada para a neuropediatria, tendo realizado uma ressonância magnética crânio-encefálica (RM-CE), que não revelou alterações. Dado o bom desenvolvimento psicomotor e a ausência de defeito neurológico aparente teve alta desta consulta.

Em reavaliação subsequente, aos cinco meses e 10 dias de idade, manteve o quadro clínico anteriormente descrito, apresentando por esta altura reflexo da convergência, cuja estimulação provocava uma diminuição da intensidade do nistagmo.

O nistagmo infantil idiopático (NII) é uma condição benigna, não sendo expectáveis outras doenças de base ou uma diminuição importante da acuidade visual. ${ }^{5}$ No entanto, atendendo à sua cronicidade, foi sugerido pelo oftalmologista que a criança mantivesse seguimento nessa especialidade. Irá manter igualmente vigilância nas consultas de SIJ do centro de saúde, de acordo com o Programa Nacional de Saúde Infantil e Juvenil (PNSIJ) em vigor. ${ }^{15}$ Será tida em atenção a evolução do nistagmo e eventuais modificações nas características deste, bem como semiologia que possa sugerir a existência de alterações da acuidade visual ou de comorbilidades potencialmente comprometedoras do normal desenvolvimento da criança.

\section{COMENTÁRIO}

Uma vez que o nistagmo, nomeadamente a forma infantil, é uma condição relativamente incomum, sendo pouco frequente na prática clínica nos cuidados de saúde primários e, geralmente, pouco abordado em termos académicos, a descrição deste caso reveste-se de uma importância considerável.

Dada a facilidade com que esta entidade pode passar despercebida e a frequente ausência de sintomatologia associada percetível para terceiros, é necessário estar desperto para a mesma durante as consultas de SIJ, principalmente nos primeiros meses de vida, quando a forma infantil do nistagmo habitualmente se manifesta. É, por isso, essencial a realização de um exame objetivo completo, que inclua uma correta avaliação oftalmológica de acordo com as indicações constantes 
no PNSIJ e no manual BoAs Práticas em Oftalmologia: ELEMENTOS CLÍNICOS DE AVALIAÇÃO E REFERENCIAÇÃO. ${ }^{16}$ Uma avaliação adequada, que compreenda uma anamnese cuidada, um exame ocular externo e dos meios transparentes do olho, da capacidade visual e dos movimentos oculares e equilíbrio oculomotor, deve fazer parte do exame oftalmológico de rotina realizado nas consultas de SIJ. Deve ser dado particular enfoque às idades-chave e a recém-nascidos pertencentes a grupos de risco, devidamente identificados nos documentos supracitados (doenças sistémicas ou condições de morbilidade, história familiar de patologia oftalmológica e sinais e/ ou sintomas oftalmológicos). Dado o tempo limitado para a realização da consulta e a necessidade de uma abordagem holística da criança, segundo um modelo biopsicossocial, poderá por vezes haver a tendência para recorrer a determinados «atalhos», assumindo-se precipitadamente a ausência de patologia de determinado sistema sem que seja feita uma avaliação dirigida do mesmo. É importante procurar contrariar esta propensão, algo que poderá ser facilitado por uma sistematização da observação a realizar e, previsivelmente, pela experiência e regularidade da prática clínica.

A descrição deste caso pretende também sensibilizar para a necessidade de investigação adicional sempre que se constate esta condição. Como referido anteriormente, o nistagmo infantil pode ser idiopático, mas outros tipos assumem um caráter mais patológico, associando-se a diferentes comorbilidades. ${ }^{5} \mathrm{Na}$ Tabela 2 encontram-se identificados os vários tipos de nistagmo infantil, bem como algumas das suas principais características. ${ }^{1,-5-5,6,8-9,11-13,17-19}$ De notar que a classificação do nistagmo infantil não é uniforme na bibliografia consultada. Assim, optou-se por uma categorização relativamente simples que evidencia o substrato patológico, isto é, o conjunto de patologias que se associam ao nistagmo, ainda que possam não constituir causa direta do mesmo. É importante ter em atenção que não há padrões de nistagmo patognomónicos. ${ }^{7}$ Vários tipos de nistagmo podem coexistir no mesmo indivíduo $^{4} \mathrm{e}$ determinadas comorbilidades são comuns a diferentes patologias (e.g., alterações oftalmológicas podem associar-se frequentemente ao albinismo, patologia neurológica ou doenças endócrinas).5,20

Um diagnóstico de nistagmo infantil de novo durante a consulta implica, primeiro que tudo, uma avaliação mais detalhada dos movimentos oculares - tipo de oscilação predominante (sacádica ou pendular), planos de oscilação, monocular ou binocular, conjugação, simetria, amplitude e frequência destes movimentos, continuidade ou periodicidade do nistagmo, influência da fixação e da convergência ocular na oculomotricidade e posição preferencial da cabeça da criança. Sabe-se que os casos de NII são tipicamente bilaterais, conjugados e horizontais, embora as oscilações possam menos frequentemente ocorrer segundo um plano torsional ou, raramente, vertical. ${ }^{5,21}$ Classicamente agravam com a fixação do olhar e melhoram com a convergência do mesmo. ${ }^{1}$ Está também descrita a existência de uma zona nula - determinada posição do olhar segundo a qual a oculomotricidade é reduzida -, o que contribui para que a criança muitas vezes assuma uma posição cefálica atípica, promovendo uma maior estabilidade ocular e consequente melhoria da acuidade visual. ${ }^{5}$

Igualmente importante é verificar, dentro do possível, eventuais alterações da anatomia externa do olho, dos meios transparentes (importante avaliação do reflexo vermelho do olho), da capacidade visual (nomeadamente recorrendo aos reflexos fotomotores e da fixação e perseguição) e da posição do olhar e equilíbrio oculomotor (teste de Hirschberg e cover test). De notar que até aos seis meses pode não haver ainda uma visão binocular eficaz, ${ }^{15}$ pelo que estes últimos testes terão as suas limitações.

A colheita de uma anamnese mais detalhada, tentando perceber quando e em que circunstâncias os pais/familiares terão percecionado o aparecimento dos movimentos oculares, bem como de uma história familiar com especial enfoque na patologia oftalmológica, são informações igualmente relevantes perante um caso de nistagmo. Embora as idades referidas na bibliografia não sejam totalmente consensuais, é geralmente aceite que a forma idiopática do nistagmo infantil surge habitualmente nos primeiros meses de vida (geralmente até às 12 semanas), não sendo típica a sua presença logo aquando do nascimento. Por sua vez, um aparecimento tardio, principalmente após os seis meses de idade, está habitualmente associado a uma forma adquirida do nistagmo, normalmente de caráter patológico. ${ }^{1,5,9}$

Ainda que o NII ocorra frequentemente de forma esporádica, há muitas vezes uma história familiar de 


\begin{tabular}{|c|c|c|}
\hline & \multicolumn{2}{|c|}{ Tipos de nistagmo infantil } \\
\hline & Idiopático & Associado a défice visual (nistagmo sensorial) \\
\hline Idade de início & Tipicamente 3-4 meses; raro ao nascimento & Tipicamente 3-4 meses \\
\hline $\begin{array}{l}\text { Substrato } \\
\text { patológico }\end{array}$ & $\begin{array}{l}\text { Sem evidência de patologia ocular, neurológica ou } \\
\text { sistémica* } \\
\text { Esporádico ou hereditário, normalmente ligado ao X } \\
\text { (gene FRMD7) }\end{array}$ & $\begin{array}{l}\text { Associado a patologia ocular ou da via visual (e.g., } \\
\text { cataratas congénitas, retinopatia da prematuridade, } \\
\text { distrofias retinianas, HNO) } \\
\text { Tipo de nistagmo infantil mais frequente }\end{array}$ \\
\hline $\begin{array}{l}\text { Características } \\
\text { clínicas }\end{array}$ & $\begin{array}{l}\text { Bilateral, conjugado, horizontal (por vezes vertical ou } \\
\text { torsional), sacádico ou pendular } \\
\uparrow \text { com a fixação e } \downarrow \text { com a convergência } \\
\text { Zona nula } \rightarrow \text { posição anómala da cabeça } \\
\text { Habitualmente ligeira } \downarrow \text { AV }\end{array}$ & $\begin{array}{l}\text { Oculomotricidade semelhante ao NII } \\
\text { AV menor que na forma idiopática dada a patologia } \\
\text { de base }\end{array}$ \\
\hline \multirow[t]{2}{*}{ Evolução } & Nistagmo e AV tipicamente melhoram & Pode melhorar; formas mais graves persistem \\
\hline & Associado ao albinismo & Latente/manifesto-latente \\
\hline Idade de início & Tipicamente 3-4 meses & Tipicamente 3-4 meses \\
\hline $\begin{array}{l}\text { Substrato } \\
\text { patológico }\end{array}$ & $\begin{array}{l}\text { Albinismo oculocutâneo ou apenas ocular } \\
\text { Associado a malformações do olho e da via visual } \\
\text { (hipoplasia da mácula a que mais } \downarrow \text { AV) }\end{array}$ & $\begin{array}{l}\text { Fortemente associado a estrabismo (tipicamente } \\
\text { convergente). Normalmente benigno. } \\
\text { Também em casos de visão monocular }\end{array}$ \\
\hline $\begin{array}{l}\text { Características } \\
\text { clínicas }\end{array}$ & $\begin{array}{l}\text { Oculomotricidade semelhante ao NII } \\
\text { Fotossensibilidade é comum } \\
\text { AV } \downarrow \downarrow \text { e visão estereoscópica } \downarrow \text {; frequente associação } \\
\text { a estrabismo }\end{array}$ & $\begin{array}{l}\text { Surge/aumenta com a oclusão de um olho } \\
\text { Bilateral, conjugado, horizontal, sacádico, fase rápida } \\
\text { no sentido do olho fixador } \\
\downarrow \text { com adução do olho fixador } \\
\text { Tipicamente sem } \downarrow \text { AV }\end{array}$ \\
\hline Evolução & Persistência do nistagmo e do défice de AV & Melhora com o tratamento do estrabismo \\
\hline
\end{tabular}

nistagmo. Existem diferentes padrões de hereditariedade descritos, sendo o mais comum o ligado ao $\mathrm{X}^{1,5,17}$

É fundamental ter em conta que qualquer diagnóstico de nistagmo na criança implica sempre referenciação para a oftalmologia para exclusão de um eventual defeito visual..$^{1,21}$

Diferentes sinais e sintomas podem sugerir uma causa não idiopática para o nistagmo (Tabela 1). Quando presentes, e na suspeita de uma etiologia não estritamente oftalmológica ou na hipótese de doenças meta- bólicas ou de patologia hereditária, justifica-se uma investigação neurológica mais detalhada, nomeadamente com recurso a RM-CE, e um eventual estudo laboratorial e/ou genético. ${ }^{1,5}$

No contexto supracitado, é opinião dos autores que a criança deverá ser referenciada à especialidade de neurologia e/ou outras consideradas pertinentes. Esta referenciação não se reveste de um caráter de exclusividade. Por exemplo, no presente caso clínico, a criança foi avaliada pela neuropediatria, ainda que não 
TABELA 2. Tipos de nistagmo infantil (continuação)

Tipos de nistagmo infantil

\begin{tabular}{|c|c|c|}
\hline & Spasmus nutans & Alterações neurológicas/metabólicas \\
\hline Idade de início & 6 meses a 3 anos $^{\dagger}$ & Variável $^{*}$ \\
\hline $\begin{array}{l}\text { Substrato } \\
\text { etiológico }\end{array}$ & $\begin{array}{l}\text { Tipicamente benigno } \\
\text { Pode estar associado a estrabismo, ambliopia, } \\
\text { problemas neurológicos ou oculares }\end{array}$ & $\begin{array}{l}\text { Causas diversas (e.g., malformações congénitas, } \\
\text { leucodistrofias, hidrocefalia, ataxia espinocerebelosa, } \\
\text { doenças metabólicas, sequelas neurológicas da } \\
\text { gravidez e parto, múltiplas síndromas genéticas) }\end{array}$ \\
\hline $\begin{array}{l}\text { Características } \\
\text { clínicas }\end{array}$ & $\begin{array}{l}\text { Tríade nistagmo + torcicolo + movimentos rítmicos } \\
\text { da cabeça } \\
\text { Intermitente, desconjugado, alta frequência e baixa } \\
\text { amplitude, pendular, horizontal, vertical ou torsional } \\
\text { Tipicamente sem défice sensorial }\end{array}$ & $\begin{array}{l}\text { Nistagmo atípico (e.g., vertical, em dente de serra, } \\
\text { alternante periódico, alta frequência), desconjugado, } \\
\text { assimétrico e/ou unilateral } \\
\text { Pode haver sintomas neurológicos (e.g., vertigens, } \\
\text { náuseas, vómitos, cefaleias, convulsões, ataxia) } \\
\text { Pode haver atraso do desenvolvimento }\end{array}$ \\
\hline Evolução & Reverte nos primeiros anos de vida & $\begin{array}{l}\text { Evolução e tratamento dependentes da causa } \\
\text { subjacente }\end{array}$ \\
\hline
\end{tabular}

Abreviaturas: $\mathrm{AV}=$ acuidade visual; $\mathrm{HNO}=$ hipoplasia do nervo ótico; $\mathrm{NII}=$ nistagmo infantil idiopático.

* NII é um diagnóstico de exclusão. Considerar que uma investigação etiológica inadequada pode culminar numa categorização errada do nistagmo como "idiopático". ${ }^{11}$

† As idades de aparecimento e remissão do spasmus nutans referidas na bibliografia consultada não são consensuais, sendo muito variável e por vezes vago o período temporal indicado. Apresenta-se uma idade de aparecimento aproximada aos vários valores apurados.

¥ Aparecimento após os 4-6 meses sugere uma forma adquirida do nistagmo. ${ }^{1,9}$

apresentasse nenhum dos sinais de alarme mencionados. O encaminhamento para outras especialidades que não a oftalmologia deve obviamente ser considerado caso a caso, tendo em conta a história clínica, semiologia, opinião do médico e senso clínico, bem como a vontade dos pais, já que a insegurança associada à incerteza relativamente à eventual coexistência de outras patologias de base pode ter implicações significativas no bem-estar da família.

O diagnóstico de nistagmo numa criança é habitualmente um fator de preocupação e ansiedade para os familiares. É, assim, dever do médico de família saber tranquilizá-los, informando-os de que esta condição raramente se associa a patologias que comportem risco de vida. Ainda assim, deve ser reforçada a necessidade de uma avaliação oftalmológica especializada, dada a possibilidade da existência de défices visuais que facilmente podem passar despercebidos, nomeadamente numa fase mais precoce da vida da criança.
Particularizando em relação ao caso clínico apresentado, o NII é considerado benigno, dado não estar associado a patologia visual ou neurológica de base. A acuidade visual nestes casos é relativamente boa, ainda que possa haver algum défice associado à oscilação ocular que condiciona uma diminuição do período de foveação, isto é, o tempo durante o qual a imagem se encontra estabilizada na retina. ${ }^{7,17}$ Curiosamente, quando o nistagmo surge na infância precoce, o indivíduo tipicamente não chega a desenvolver oscilópsia (perceção de imagem oscilatória), provavelmente através de mecanismos de adaptação cerebral à oculomotricidade mantida. ${ }^{1,9}$ Apesar de ser uma condição crónica, existe tendência para que os movimentos oculares anormais diminuam de intensidade com a idade..$^{5,9,21} \mathrm{~A}$ acuidade visual da criança vai também aumentando com o seu desenvolvimento, em linha com o que é habitual na população geral, embora cerca de metade das crianças venha a ter uma visão ligeiramente abaixo dos limiares 
considerados normais. ${ }^{7,18}$ Na maioria dos casos, a visão binocular (estereopsia) está mantida..$^{5,17}$

Nos casos de nistagmo infantil associado a patologia oftalmológica, a acuidade visual é normalmente inferior ${ }^{1,7,18} \mathrm{e}$ a evolução clínica pode ser variável, dependendo da condição de base. ${ }^{19}$ Também no nistagmo associado ao albinismo a acuidade visual é inferior, ${ }^{6-7,18}$ enquanto nos casos de nistagmo latente/manifesto-latente ou spasmus nutans a acuidade visual está habitualmente mantida. ${ }^{13}$

O nistagmo infantil não tem cura, sendo que os tratamentos atualmente disponíveis podem reduzir os movimentos oculares anormais e/ou melhorar a acuidade visual, mas não é expectável uma remissão total da sintomatologia. As intervenções terapêuticas devem ser implementadas precocemente, de forma a potenciar o máximo benefício, promovendo o desenvolvimento e maturação adequados do sistema visual. ${ }^{22} \mathrm{~A}$ correção dos erros refrativos, frequentemente associados ao nistagmo infantil, é talvez a intervenção mais importante para a melhoria da acuidade visual, potencialmente reduzindo a incidência de ambliopia refrativa. ${ }^{9,18,23}$ Outras abordagens terapêuticas incluem a correção de um eventual estrabismo (oclusão ocular, lentes prismáticas ou cirurgia, por exemplo), a utilização de prismas divergentes no sentido de forçar uma convergência ocular, que pode nalguns casos reduzir a oculomotricidade, e o recurso à cirurgia para reposicionar a zona nula na posição primária do olhar, reduzindo o nistagmo e promovendo a adoção de uma posição cefálica anatomicamente normal. Alguns fármacos (memantina, gabapentina e baclofeno) podem ter efeito benéfico no nistagmo, embora sejam necessários mais estudos, nomeadamente na população infantil. O uso de toxina botulínica poderá eventualmente ter um papel na redução da oculomotricidade sendo, no entanto, mal tolerado. ${ }^{1,5,9}$

Verifica-se, assim, que, de entre as várias intervenções terapêuticas disponíveis, ou que têm ainda um estatuto muito experimental, são necessários mais estudos para a sua implementação regular na população pediátrica ou estão fora do âmbito de atuação clínica do médico de família, necessitando de uma abordagem médica e/ou cirúrgica pela especialidade de oftalmologia. No entanto, considera-se que existem determinadas medidas que devem ser adotadas nas consultas de Medicina Geral e Familiar e que se revestem de uma importância fundamental.

Em primeiro lugar, e como referido anteriormente, qualquer caso de nistagmo infantil deverá ser devidamente descrito e referenciado para a especialidade de oftalmologia. Se houver indicação para que a criança mantenha seguimento em consulta/s da/s especialidade/s, é papel do médico de família, enquanto profissional de saúde com um contacto próximo do doente e da sua família, acompanhando-os ao longo de todo o percurso clínico, verificar que a criança tem de facto tido o seguimento recomendado, eventualmente servindo de interface e de «ponto-de-encontro» entre as diferentes especialidades, integrando toda a informação clínica e orientando corretamente a criança e sua família consoante as necessidades.

Seguidamente, e porque o nistagmo, ainda que idiopático, se pode associar com alguma frequência a défice da acuidade visual, destaque-se a importância de uma avaliação regular da visão nas consultas de SIJ. Os familiares devem igualmente ser questionados relativamente à suspeita de eventuais alterações visuais. É também importante ir avaliando o nistagmo ao longo do tempo, verificando a sua evolução (tendência a diminuir com a idade, caso seja idiopático) e vigiando eventuais alterações nas suas características. $\mathrm{O}$ aparecimento de novos padrões na oculomotricidade, especialmente se atípicos (alteração dos planos de oscilação ocular, principalmente se vertical, assimetria entre os olhos, aumento da frequência do nistagmo ou a presença de movimentos oculares caóticos), deve constituir um motivo de preocupação e exige uma avaliação oftalmológica especializada atempada.

Beneficiando de uma posição privilegiada no acompanhamento das crianças ao longo do tempo, dada a regularidade das consultas de SIJ, principalmente nos primeiros anos de vida, o médico de família tem assim um papel primordial na vigilância do seu crescimento. É importante estar atento a eventuais atrasos no desenvolvimento psicomotor ou na evolução estaturo-ponderal, não descurando o uso regular, como é recomendado, da escala de desenvolvimento de Mary Sheridan modificada e das curvas de crescimento da Organização Mundial da Saúde. Alterações significativas no padrão habitual de desenvolvimento da criança ou o aparecimento de semiologia neurológica anormal (e.g., reflexos 
atípicos, desequilíbrio da marcha, hipotonia) constituem um sinal de preocupação e podem estar inerentes a patologia neurológica, metabólica e/ou genética de base, que não tenha sido previamente identificada e correlacionada com o nistagmo. Ainda assim, e como se verifica, não é de esperar uma abordagem significativamente diferente da restante população pediátrica-uma vigilância regular e uma avaliação global e sistematizada é adequada ao acompanhamento destas crianças pelo médico assistente.

Por último, de destacar a abordagem holística que o médico de família deve ter perante o doente. Assim, numa primeira fase, é preciso estar atento às implicações que o diagnóstico de nistagmo numa criança pode ter na família. Como anteriormente referido, o médico deve certificar-se de que os pais (ou outros familiares cuidadores) compreendem esta entidade, as possíveis causas, consequências e prognóstico. Deve ser avaliado o nível de ansiedade e preocupação dos familiares, sabendo gerir corretamente a situação do ponto de vista científico, clínico e humano.

Posteriormente, em crianças de maior idade, as consequências sociais do nistagmo devem ser tidas em conta. Há evidência de que, para além das implicações na acuidade visual, o nistagmo pode ter repercussões na funcionalidade social dos indivíduos. ${ }^{24} \mathrm{~A}$ presença desta condição numa criança pode afetar negativamente o seu bem-estar emocional, nomeadamente pela preocupação com a aparência e autoconfiança e pelo risco de ser gozada pelos colegas ou sofrer bullying. ${ }^{24}$ Esta é também uma vertente com que o médico de família se deve preocupar, questionando pais e crianças relativamente ao seu estado emocional e relação com os pares, procurando apurar eventuais sinais de alarme e fatores de stresse cuja abordagem é igualmente importante.

Concluindo, o médico de família, dado o contacto próximo e continuado que tem com as crianças da sua lista de utentes, muitas vezes desde o período pré-natal, assume uma função primordial na deteção, avaliação inicial e referenciação adequada dos casos de nistagmo infantil. Embora raramente associado a patologias que comportem risco de vida, o nistagmo pode estar associado a alterações oftalmológicas, neurológicas ou sistémicas, pelo que o seu diagnóstico e investigação etiológica precoces são fundamentais. É, assim, essencial que o médico esteja atento a eventuais anomalias da motricidade ocular objetiváveis durante as consultas de SIJ e saiba encaminhar devidamente o doente para uma investigação atempada, vigiando a evolução clínica do quadro e estando atento a sinais de alarme. Uma vez que esta entidade pode facilmente despoletar ansiedade nos pais ou familiares da criança, é importante esclarecê-los e tranquilizá-los de forma sensata, evitando comunicar prognósticos precoces e precipitados e procurando limitar o surgimento de falsas expectativas ou de eventuais instabilidades na dinâmica familiar.

\section{AGRADECIMENTOS}

Os autores agradecem à Dr. ${ }^{a}$ Margarida Fazio, diretora do internato médico de medicina geral e familiar do ACeS Lisboa Norte, pelos comentários e sugestões gentilmente prestados durante a elaboração do manuscrito.

\section{REFERÊNCIAS BIBLIOGRÁFICAS}

1. Ehrt O. Infantile and acquired nystagmus in childhood. Eur J Paediatr Neurol. 2012;16(6):567-72.

2. Barton JJ. Overview of nystagmus. UpToDate [Internet]; 2017 Jun [updated 2019 May 29; cited 2019 Feb 2]. Available from: https://www.uptodate.com/contents/overview-of-nystagmus

3. Theodorou M, Clement R. Classification of infantile nystagmus waveforms. Vision Res. 2016;123:20-5.

4. Abadi RV. Mechanisms underlying nystagmus. J R Soc Med. 2002;95 (5):231-4

5. Papageorgiou E, McLean RJ, Gottlob I. Nystagmus in childhood. Pediatr Neonatol. 2014;55(5):341-51.

6. Mravicic I, Lukacevic S, Bohac M, Pauk-Gulic M, Glavota V. Nystagmus. In: Mravicic I, editor. Eye motility. IntechOpen; 2019 [cited 2019 Nov 16]. chapter 4.Available from: https://www.intechopen.com/books/eyemotility/nystagmus

7. Abadi RV, Bjerre A. Motor and sensory characteristics of infantile nystagmus. Br J Ophthalmol. 2002;86(10):1152-60.

8. Sarvananthan N, Surendran M, Roberts EO, Jain S, Thomas S, Shah N, et al. The prevalence of nystagmus: the Leicestershire Nystagmus Survey. Invest Ophthalmol Vis Sci. 2009;50(11):5201-6.

9. Atilla $\mathrm{H}$. Assessment and management of infantile nystagmus syndrome. J Clin Exp Ophthalmol. 2016;7(2):1000550.

10. Nash DL, Diehl NN, Mohney BG. Incidence and types of pediatric nystagmus. Am J Ophthalmol. 2017;182:31-4.

11. Bertsch M, Floyd M, Kehoe T, Pfeifer W, Drack AV. The clinical evaluation of infantile nystagmus: what to do first and why. Ophthalmic Genet. 2017;38(1):22-33.

12. Richards MD, Wong A. Infantile nystagmus syndrome: clinical characteristics, current theories of pathogenesis, diagnosis, and management. Can J Ophthalmol. 2015;50(6):400-8.

13. CEMAS Working Group. A National Eye Institute sponsored workshop and publication on the Classification of Eye Movement Abnormalities 
and Strabismus (CEMAS). Bethesda, MD: The National Eye Institute; 2001.

14. Hertle RW. Nystagmus and ocular oscillations in infancy and childhood. In: Wright KW, Spiegel PH, editors. Pediatric ophthalmology and strabismus. New York: Springer; 2003. p. 954-69. ISBN 9780387217536

15. Direcção-Geral da Saúde. (2013). Programa nacional de saúde infantil e juvenil: circular normativa n. ${ }^{\circ}$ 010/2013, de 31/05/2013. Lisboa: DGS; 2013.

16. Direcção de Serviços de Cuidados de Saúde, Comissão de Coordenação do Programa Nacional para a Saúde da Visão. Boas práticas em oftalmologia 2008: elementos clínicos de avaliação e referenciação [Internet]. Lisboa: Direção-Geral da Saúde; 2008. Available from: http://nocs.pt/wp-content/uploads/2016/04/Boas-Praticas-em-Oftalmologia.pdf

17. Thomas S, Proudlock FA, Sarvananthan N, Roberts EO, Awan M, McLean $R$, et al. Phenotypical characteristics of idiopathic infantile nystagmus with and without mutations in FRMD7. Brain. 2008;131(Pt 5):125967.

18. Fu VL, Bilonick RA, Felius J, Hertle RW, Birch EE. Visual acuity development of children with infantile nystagmus syndrome. Invest Ophthalmol Vis Sci. 2011;52(3):1404-11.

19. Buncic JR. A clinician's approach to infantile nystagmus. Am Orthopt J. 2004;54:88-94.
20. Poll-The BT, Wenniger-Prick CJ. The eye in metabolic diseases: clues to diagnosis. Eur J Paediatr Neurol. 2011;15(3):197-204.

21. Barton JJ. Pendular nystagmus. UpToDate [Internet]; 2018 Dec [updated 2018 Dec 6; cited 2019 Feb 2]. Available from: https://www.uptodate.com/contents/pendular-nystagmus

22. Atilla H, Demir HD, IşıkçelikY. Long-term results of four horizontal rectus muscle recession in nystagmus treatment. Strabismus. 2014;22(2): 81-5.

23. Hertle RW. Examination and refractive management of patients with nystagmus. Surv Ophthalmol. 2000;45(3):215-22.

24. Pilling RF, Thompson JR, Gottlob I. Social and visual function in nystagmus. Br J Ophthalmol. 2005;89(10):1278-81.

\section{CONFLITO DE INTERESSES}

Os autores declaram não ter quaisquer conflitos de interesse.

\section{ENDEREÇO PARA CORRESPONDÊNCIA}

Elmano Margato

E-mail: elmanomargato@campus.ul.pt

https://orcid.org/0000-0003-2606-1277

Recebido em 27-05-2019

Aceite para publicação em 22-12-2019

\section{ABSTRACT}

\section{INFANTILE NYSTAGMUS: AN INNOCUOUS CONDITION OR SOMETHING ELSE? BASED ON A CASE REPORT}

Introduction: Infantile nystagmus may or may not be associated with visual and/or neurological disorders and constitutes an uncommon and rarely observed entity in Family Medicine clinical practice. We sought to find out how the family physician should act before this condition.

Case description: We describe the case of an infant with no relevant personal or family history in whom the family physician, during the preventive care visit at four months of age, identified the presence of a bilateral, conjugate, symmetrical, horizontal and continuous nystagmus. Based on a quick referral to pediatric Ophthalmology and Neurology, the existence of a concomitant neurological condition was excluded. The child was followed up by the ophthalmologist and family physician, monitoring his progress according to current recommendations.

Comment: This case report demonstrates the importance of a systematic and comprehensive assessment of children at preventive care visits. A thorough physical examination should not be neglected, avoiding hasty assumptions. It also reinforces the need for adequate ophthalmic evaluation, particularly in the first months of life - when infantile nystagmus usually manifests -, as well as the importance of further investigation into this condition. Although many of these cases occur in an isolated manner, most seem to be associated with visual defects, with the family physician having a primary role in the identification, referral, and follow-up of these children, being aware of possible disturbances in their development and socialization, as well as consequences on the family dynamics and stability.

Keywords: Nystagmus, Congenital; Nystagmus, Pathologic; Family physician. 